\title{
Metacomputing Revisited: Alternative Paradigms for Distributed Resource Sharing
}

\author{
Vaidy Sunderam \\ Department of Math \& Computer Science \\ Emory University, Atlanta, GA 30322, USA \\ vss@emory. edu \\ http: / /www.mathcs. emory. edu/dcl/
}

\begin{abstract}
Conventional distributed computing paradigms such as PVM and $\operatorname{MPI}(\mathrm{CH})$ have had mixed success when translated to computing environments that span multiple administrative and ownership domains. We analyze fundamental issues in distributed resource sharing particularly from the viewpoint of different forms of heterogeneity - i.e. not just in the computing platforms, but also in storage, network characteristics, availability, access protocols, robustness, and dynamicity. We argue that effective multidomain resource sharing in the face of such variability is critically dependent upon minimizing global state between providers, and between providers and clients. The $\mathrm{H} 2 \mathrm{O}$ framework has made one step in this direction, by decoupling provider concerns from client requirements, and enabling clients to (re)configure resources as required. $\mathrm{H} 2 \mathrm{O}$ is based on a "pluggable" software architecture to enable flexible and reconfigurable distributed computing. A key feature is the provisioning of customization capabilities that permit clients to tailor provider resources as appropriate to the given application, without compromising control or security. Through the use of uploadable "pluglets", users can exploit specialized features of the underlying resource, application libraries, or optimized message passing subsystems on demand. The next generation of this framework takes the second step, to virtualize and homogenize resource aggregates at the client side, thereby further reducing the degree of coupling between providers, and between providers and clients. The system also supports dynamic environment preconditioning to automate many of the tasks required in multidomain resource sharing. The architecture and design philosophies of these software frameworks, their implementation, recent experiences, and planned enhancements are described, in the context of new paradigms and directions for metacomputing.
\end{abstract}

